\title{
Output Feedback Stabilization for Stochastic Nonholonomic Systems under Arbitrary Switching
}

\author{
Xianjun Liu, ${ }^{1}$ Yinghui Zhao, ${ }^{2}$ Ye Yue, ${ }^{2}$ and Dongkai Zhang ${ }^{2}$ \\ ${ }^{1}$ College of Mathematics and Information Science, Hebei Normal University, Shijiazhuang 050024, China \\ ${ }^{2}$ School of Science, Shijiazhuang University, Shijiazhuang 050035, China \\ Correspondence should be addressed to Dongkai Zhang; zdkmailhot@126.com
}

Received 2 August 2017; Revised 28 October 2017; Accepted 6 November 2017; Published 29 November 2017

Academic Editor: Zhengqiu Zhang

Copyright ( 2017 Xianjun Liu et al. This is an open access article distributed under the Creative Commons Attribution License, which permits unrestricted use, distribution, and reproduction in any medium, provided the original work is properly cited.

The output feedback controllers of stochastic nonholonomic systems under arbitrary switching are discussed. We adopt an observer which can simplify the design process. The designed control laws cause the calculation of the gain parameter to be very convenient since the denominator of virtual controllers does not contain the gain parameter. Finally, an example is given to show the effectiveness of controllers.

\section{Introduction}

In recent years, switched system's control, especially under arbitrary switching, has become an active field [1-3]. The global stabilization of switched systems based on arbitrary switching was given [4-6]. The stabilizing controllers of switched systems with arbitrary switching were given [7-10]. The output feedback controllers of nonlinear systems with arbitrary switching were designed [11, 12].

In the past ten years, the problem of stabilization for stochastic nonholonomic systems (SNSs) received much attention. They mainly can be classified into two types. The first is state-feedback control: stabilization [13, 14], adaptive stabilization [15-18], finite-time stabilization [19], stabilization with time-varying delays [20], and stabilization of mobile robots [21,22]. The second is the output feedback stabilization [23-25].

Since sometimes part of the system states are unmeasurable, output feedback controllers are needed. Zhang et al. discussed the output feedback stabilizing controllers of SNSs whose virtual control $b_{i}$ contains gain parameter $L$ [25]. This will lead to a problem where the calculation of $L$ is very difficult, especially for $n \geq 3$, since the inequalities about $L$ were quintic. In addition, to the authors' knowledge, there are some results about state-feedback stabilization of SNSs with Markovian switching [13, 14], with few available results for the output stabilization of SNSs under arbitrary switching. Based on the above analysis, there exists a problem, that is, how to choose a proper observer under arbitrary switching where the virtual control $b_{i}$ in controllers does not contain gain parameter $L$, which causes the calculation of $L$ to be easier.

Notations. $\mathbb{R}$ denotes the set of all real numbers. $\mathbb{R}^{n}$ denotes the real $n$-dimensional space. For a vector or matrix $X \in$ $\mathbb{R}^{n \times m}, X^{T}$ denotes its transpose, $\|X\|$ denotes the Euclidean norm, $\operatorname{Tr}\{X\}$ is its trace when $X$ is square, and $\mathscr{L}$ is a stochastic differential operator [26].

\section{Problem Formulation}

The stochastic nonholonomic nonlinear systems are given by

$$
\begin{aligned}
\mathrm{d} x_{0}= & u_{0} \mathrm{~d} t+g_{[0, \sigma(t)]}^{T}\left(x_{0}\right) \mathrm{d} \omega, \\
\mathrm{d} x_{i}= & u_{0} x_{i+1} \mathrm{~d} t+f_{[i, \sigma(t)]}\left(x_{0}, \bar{x}_{i}\right) \mathrm{d} t \\
& +g_{[i, \sigma(t)]}^{T}\left(x_{0}, \bar{x}_{i}\right) \mathrm{d} \omega, \quad i=1, \ldots, n-1, \\
\mathrm{~d} x_{n}= & u \mathrm{~d} t+f_{[n, \sigma(t)]}\left(x_{0}, x\right) \mathrm{d} t+g_{[i, \sigma(t)]}^{T}\left(x_{0}, x\right) \mathrm{d} \omega, \\
y= & {\left[x_{0}, x_{1}\right]^{T}, }
\end{aligned}
$$


where $u_{0}$ and $u \in \mathbb{R}$ are inputs, $x_{0} \in \mathbb{R}$ and $\left(x_{1}, \ldots, x_{n}\right)^{T} \in \mathbb{R}^{n}$ are system states, $\bar{x}_{i}=\left(x_{1}, \ldots, x_{i}\right)^{T}, f_{i} \triangleq f_{i}\left(x_{0}, \bar{x}_{i}\right): \mathbb{R}^{i+1} \rightarrow$ $\mathbb{R}$ are smooth functions named as nonlinear drifts with $f_{i}(0,0)=0$, and $g_{0}\left(x_{0}\right): \mathbb{R} \rightarrow \mathbb{R}^{m}$ and $g_{i} \triangleq g_{i}\left(x_{0}, \bar{x}_{i}\right):$ $\mathbb{R}^{i+1} \rightarrow \mathbb{R}^{m}$ are smooth functions with $g_{0}(0)=0$ and $g_{i}(0,0)=0,1 \leq i \leq n . \sigma(t):[0,+\infty) \rightarrow M=\{1,2, \ldots, m\}$ is a piecewise constant switching signal, and $\omega \in \mathbb{R}^{m}$ is an $m$-dimensional independent standard Wiener process.

Assumption 1. If there exist smooth function $v_{[0, \sigma(t)]}\left(x_{0}\right)$ : $\mathbb{R} \rightarrow \mathbb{R}^{m}$ and positive constant $\bar{m}$, then

$$
\begin{aligned}
g_{[0, \sigma(t)]}\left(x_{0}\right) & =x_{0} v_{[0, \sigma(t)]}\left(x_{0}\right), \\
\left\|\nu_{[0, \sigma(t)]}\left(x_{0}\right)\right\| & \leq \bar{m},
\end{aligned}
$$

where $v_{[0, \sigma(t)]}\left(x_{0}\right)$ is a known bounded nonnegative function.

Assumption 2. For $i=1, \ldots, n$, one has known constants $\bar{\nu}_{[i, \sigma(t)]}$ and $\bar{\gamma}_{[i, \sigma(t)]}$ satisfying

$$
\begin{aligned}
\left|f_{[i, \sigma(t)]}\left(x_{0}, \bar{x}_{i}\right)\right| & \leq\left(\left|x_{1}\right|+\cdots+\left|x_{i}\right|\right) \bar{\gamma}_{[i, \sigma(t)]}, \\
\left\|g_{[i, \sigma(t)]}\left(x_{0}, \bar{x}_{i}\right)\right\| & \leq\left(\left|x_{1}\right|+\cdots+\left|x_{i}\right|\right) \bar{\gamma}_{[i, \sigma(t)]} .
\end{aligned}
$$

Remark 3. There are two main differences between systems (1a) and (1b) and those in $[24,25]$. The first is the arbitrary switching mentioned in this paper. The second will be illustrated in Remark 7. In addition, Assumptions 1 and 2 are similar to those in $[24,25]$, but in fact they hold under arbitrary switching; for example, $\bar{\gamma}_{[i, \sigma(t)]}=\max \left\{\bar{\gamma}_{[i, 1]}, \bar{\gamma}_{[i, 2]}, \bar{\gamma}_{[i, 3]}, \bar{\gamma}_{[i, 4]}\right\}$ when $\sigma(t):[0,+\infty) \rightarrow M=\{1,2,3,4\}$.

\section{Output Feedback Stabilization}

The controller design in Sections 3.1 and 3.2 is under $x_{0}\left(t_{0}\right) \neq$ 0. The other one is discussed in Section 3.3.

3.1. Controller $u_{0}$ Design. For subsystem (1a), one obtains

$$
\mathscr{L} V_{0}\left(x_{0}\right) \leq-2 \lambda V_{0}\left(x_{0}\right) .
$$

with Lyapunov function $V_{0}\left(x_{0}\right)=(1 / 2) x_{0}^{2}$ and controller $u_{0}$ as follows:

$$
\begin{aligned}
& u_{0}=-\eta_{0} x_{0}, \\
& \eta_{0}=\lambda+\frac{1}{2} \bar{m}^{2},
\end{aligned}
$$

where $\lambda>0$ is a design real number.

Theorem 4. For system (1a), the closed-loop system with controller (4) is asymptotically stable in probability.

By (4) and (1a), one has

$$
\mathrm{d} x_{0}=-\eta_{0} x_{0} \mathrm{~d} t+g_{[0, \sigma(t)]}^{T}\left(x_{0}\right) \mathrm{d} \omega .
$$

Remark 5. For any $x_{0}\left(t_{0}\right) \neq 0$, one has $x_{0}(t) \neq 0$ in $(6)$ at the time interval $t \in\left(t_{0},+\infty\right)$ a.s. with a similar proof of Proposition 1 in [25].
3.2. Controller u Design. In order to design controller $u$, let

$$
z_{i}=\frac{x_{i}}{u_{0}^{n-i}}, \quad 1 \leq i \leq n .
$$

Remark 6. For any $x_{0}\left(t_{0}\right) \neq 0$, from Remark 5 , we have that

(7) is meaningful a.s.

By (lb) and (7), one has

$$
\begin{aligned}
& \mathrm{d} z_{i}=z_{i+1} \mathrm{~d} t+\phi_{[i, \sigma(t)]}\left(x_{0}, \bar{z}_{i}\right) \mathrm{d} t+\psi_{[i, \sigma(t)]}^{T}\left(x_{0}, \bar{z}_{i}\right) \mathrm{d} \omega, i=1, \ldots, n-1, \\
& \mathrm{~d} z_{n}=u \mathrm{~d} t+\phi_{[n, \sigma(t)]}\left(x_{0}, x\right) \mathrm{d} t+\psi_{[n, \sigma(t)]}^{T}\left(x_{0}, x\right) \mathrm{d} \omega, \\
& y_{1}=z_{1},
\end{aligned}
$$

where $\bar{z}_{i}=\left[z_{1}, \ldots, z_{i}\right]^{T}$ and

$$
\begin{aligned}
& \phi_{[i, \sigma(t)]}\left(x_{0}, \bar{z}_{i}\right) \\
& =\eta_{0}(n-i) z_{i}+\frac{f_{[i, \sigma(t)]}}{u_{0}^{n-i}} \\
& \quad+\frac{1}{2}(n-i)(n-i+1) z_{i} \frac{g_{[0, \sigma(t)]}^{T} g_{[0, \sigma(t)]}}{x_{0}^{2}} \\
& +\eta_{0}(n-i) \frac{g_{[i, \sigma(t)]}^{T} g_{[0, \sigma(t)]}}{u_{0}^{n-i+1}}, \quad i=1, \ldots, n . \\
& \psi_{[i, \sigma(t)]}\left(x_{0}, \bar{z}_{i}\right)=\frac{g_{[i, \sigma(t)]}}{u_{0}^{n-i}-(n-i) z_{i} \frac{g_{[0, \sigma(t)]}}{x_{0}},}
\end{aligned}
$$

We adopt the following observer [27]:

$$
\begin{aligned}
& \dot{\vec{z}}_{i}=\widehat{z}_{i+1}+L^{i} a_{i}\left(y_{1}-\widehat{z}_{1}\right), \quad i=1, \ldots, n-1, \\
& \dot{\vec{z}}_{n}=u+L^{n} a_{n}\left(y_{1}-\widehat{z}_{1}\right),
\end{aligned}
$$

where $L \geq 1$ is a gain parameter and real numbers $a_{i}>0$, $i=1, \ldots, n$, such that $p(s)=s^{n}+a_{1} s^{n-1}+\cdots+a_{n}$ is Hurwitz.

Remark 7. The second main difference between this manuscript and [25] is that the observer (10) we choose is the same as that in [27], but different from that in [25]. This observer has two advantages. The first is that it can simplify the process of designing controllers. The second is that $b_{i}$ in virtual control $\alpha_{i}$ that we design in the following does not contain the gain parameter $L$.

Denoting

$$
\xi_{i}=\frac{\left(z_{i}-\widehat{z}_{i}\right)}{L^{i-1}}, \quad i=1, \ldots, n,
$$

one has the error systems

$$
\mathrm{d} \xi=L A \xi \mathrm{d} t+\Phi_{\sigma(t)} \mathrm{d} t+\Psi_{\sigma(t)}^{T} \mathrm{~d} \omega,
$$


where

$$
\begin{aligned}
& A=\left[\begin{array}{cc}
-a_{1} & \\
-a_{2} & I_{n-1} \\
\vdots & \\
-a_{n} & 0
\end{array}\right], \\
& \Phi_{\sigma(t)}=\left[\begin{array}{c}
\phi_{[1, \sigma(t)]} \\
\frac{\phi_{[2, \sigma(t)]}}{L} \\
\vdots \\
\frac{\phi_{[n-1, \sigma(t)]}}{L^{n-2}} \\
\frac{\phi_{[n, \sigma(t)]}}{L^{n-1}}
\end{array}\right], \\
& \Psi_{\sigma(t)}^{T}=\left[\begin{array}{c}
\psi_{[1, \sigma(t)]}^{T} \\
\frac{\psi_{[2, \sigma(t)]}^{T}}{L} \\
\vdots \\
\frac{\psi_{[n-1, \sigma(t)]}^{T}}{L^{n-2}} \\
\frac{\psi_{[n, \sigma(t)]}^{T}}{L^{n-1}}
\end{array}\right] .
\end{aligned}
$$

Therefore, for positive definite matrix $P, A$ is a Hurwitz matrix; that is, $A^{T} P+P A=-I$. Now, one has

$$
\begin{aligned}
\mathrm{d} \xi & =L A \xi \mathrm{d} t+\Phi_{\sigma(t)} \mathrm{d} t+\Psi_{\sigma(t)}^{T} \mathrm{~d} \omega, \\
\mathrm{d} y_{1} & =z_{2} \mathrm{~d} t+\phi_{[1, \sigma(t)]} \mathrm{d} t+\psi_{[1, \sigma(t)]}^{T} \mathrm{~d} \omega, \\
\dot{\bar{z}}_{i} & =\widehat{z}_{i+1}+L^{i} a_{i}\left(y_{1}-\widehat{z}_{1}\right), \quad i=1, \ldots, n-1, \\
\dot{\bar{z}}_{n} & =u+L^{n} a_{n}\left(y_{1}-\widehat{z}_{1}\right) .
\end{aligned}
$$

Proposition 8. By Assumptions 1 and 2, there exist constants $\tau_{1}>0$ and $\tau_{2}>0$ a.s., such that

$$
\begin{aligned}
\left|\phi_{[i, \sigma(t)]}\right| & \leq\left(\left|z_{1}\right|+\left|z_{2}\right|+\cdots+\left|z_{i}\right|\right) \tau_{1}, \\
\left|\psi_{[i, \sigma(t)]}^{T}\right| & \leq\left(\left|z_{1}\right|+\left|z_{2}\right|+\cdots+\left|z_{i}\right|\right) \tau_{2}, \\
\left\|\Phi_{\sigma(t)}\left(x_{0}, z\right)\right\|^{2} & \leq n\left(\left|z_{1}\right|+\frac{\left|z_{2}\right|}{L}+\cdots+\frac{\left|z_{n}\right|}{L^{n-1}}\right)^{2} \tau_{1}^{2}, \\
\left\|\Psi_{\sigma(t)}^{T}\left(x_{0}, z\right)\right\|^{2} & \leq n\left(\left|z_{1}\right|+\frac{\left|z_{2}\right|}{L}+\cdots+\frac{\left|z_{n}\right|}{L^{n-1}}\right)^{2} \tau_{2}^{2},
\end{aligned}
$$

where $z=\left[z_{1}, \ldots, z_{n}\right]^{T}$.

Remark 9. The proof of the above proposition is similar to that of Proposition 2 in [25]. In fact, we only need to let $\overline{\bar{v}}_{i}=$ $\eta_{0}+\left(\left|\eta_{0} \bar{m}\right|^{i-1}+\cdots+1\right) \max _{\sigma(t)} \bar{v}_{[i, \sigma(t)]}$.
One can define variables $\varepsilon_{i}$ as follows in order to utilize the backstepping method:

$$
\begin{aligned}
\varepsilon_{1} & =z_{1}, \\
\varepsilon_{i}\left(z_{1}, \widehat{z}_{2}, \ldots, \widehat{z}_{i}\right) & =\widehat{z}_{i}-\alpha_{i-1}\left(z_{1}, \widehat{z}_{2}, \ldots, \widehat{z}_{i-1}\right),
\end{aligned}
$$

with virtual smooth controllers $\alpha_{i-1}(i=2, \ldots, n)$.

Step 1. For positive parameter $\delta$, letting $V_{0}=\delta \xi^{T} P \xi$, with a similar method in [24], one has

$$
\begin{aligned}
\mathscr{L} V_{0} \leq & -\left[\delta\left(L-2\|P\|^{2}\right)-2 n C_{e}\right]\|\xi\|^{2} \\
& +2 n C_{e}\left(\left|z_{1}\right|^{2}+\frac{\left|\widehat{z}_{2}\right|^{2}}{L^{2}}+\cdots+\frac{\left|\bar{z}_{2}\right|^{2}}{L^{2 n-2}}\right),
\end{aligned}
$$

where $C_{e}=n \delta \tau_{1}^{2}+n \delta \lambda_{\max }(P) \tau_{2}^{2}$. Defining $V_{1}=V_{0}+(1 / 2) \varepsilon_{1}^{2}$, we have

$$
\begin{aligned}
\mathscr{L} V_{1} \leq & -\left[\delta\left(L-2\|P\|^{2}\right)-2 n C_{e}\right]\|\xi\|^{2} \\
& +2 n C_{e}\left(\left|z_{1}\right|^{2}+\frac{\left|\widehat{z}_{2}\right|^{2}}{L^{2}}+\cdots+\frac{\left|\widehat{z}_{n}\right|^{2}}{L^{2 n-2}}\right) \\
& +\varepsilon_{1}\left[\varepsilon_{2}+\alpha_{1}+L \xi_{2}+\phi_{[1, \sigma(t)]}\right] \\
& +\frac{1}{2} \operatorname{Tr}\left[\psi_{[1, \sigma(t)]} \psi_{[1, \sigma(t)]}^{T}\right] .
\end{aligned}
$$

The following inequalities hold with Lemma 2.1 in [28]:

$$
\begin{aligned}
2 n C_{e} \frac{\left|\hat{z}_{2}\right|^{2}}{L^{2}} & \leq 4 n C_{e} \frac{\varepsilon_{2}^{2}}{L}+4 n C_{e} \frac{\alpha_{1}^{2}}{L^{2}}, \\
\varepsilon_{1} \varepsilon_{2} & \leq \frac{1}{4} L \varepsilon_{2}^{2}+L \varepsilon_{1}^{2}, \\
L \varepsilon_{1} \xi_{2} & \leq \frac{L}{4} \varepsilon_{1}^{2}+L \xi_{2}^{2}, \\
\varepsilon_{1} \phi_{[1, \sigma(t)]} & \leq L \tau_{1}^{2} \varepsilon_{1}^{2}, \\
\frac{1}{2} \operatorname{Tr}\left[\psi_{[1, \sigma(t)]} \psi_{[1, \sigma(t)]}^{T}\right] & \leq \frac{1}{2} L \tau_{2}^{2} \varepsilon_{1}^{2}, \\
2 n C_{e}\left|z_{1}\right|^{2} & \leq 2 n C_{e} L \varepsilon_{1}^{2},
\end{aligned}
$$

where, together with (18), $\left|z_{i} / L^{i-1}\right| \leq\left\|\xi_{i}\right\|+\left|\bar{z}_{i} / L^{i-1}\right|$. Choosing

$$
\begin{aligned}
& \alpha_{1}=-L b_{1} \varepsilon_{1}, \\
& b_{1}=2 n+1+2 n C_{e}+\frac{5}{4}+\tau_{1}+\frac{\tau_{2}^{2}}{2},
\end{aligned}
$$

one has

$$
\begin{aligned}
\mathscr{L} V_{1} \leq & -\left[\delta\left(L-2\|P\|^{2}\right)-2 n C_{e}-L\right]\|\xi\|^{2} \\
& +2 n C_{e}\left[\frac{\left|\widehat{z}_{3}\right|^{2}}{L^{4}}+\cdots+\frac{\left|\bar{z}_{n}\right|^{2}}{L^{2 n-2}}\right] \\
& -\left[(2 n+1) L-4 n C_{e} b_{1}^{2}\right] \varepsilon_{1}^{2}+\frac{1}{4 L} \varepsilon_{2}^{2}+4 n C_{e} \frac{\varepsilon_{2}^{2}}{L} .
\end{aligned}
$$


Step $i(2 \leq i \leq n-1)$. From (16), we have

$$
\begin{gathered}
\varepsilon_{i}=\widehat{z}_{i}+L b_{i-1} \widehat{z}_{i-1}+L^{2} b_{i-1} b_{i-2} \widehat{z}_{i-2}+\cdots+L^{i-1} b_{i-1} b_{i-2} \cdots b_{1} z_{1}, \\
\mathrm{~d} \varepsilon_{i}=\left[\widehat{z}_{i+1}+L^{i} \widetilde{d}_{i} \xi_{1}+L^{i} d_{i, 1} \varepsilon_{1}+L^{i-1} d_{i, 2} \varepsilon_{2}+\cdots+L^{2} d_{i, i-1} \varepsilon_{i-1}+L d_{i, i} \varepsilon_{i}\right] \mathrm{d} t \\
+L^{i-1} b_{i-1} b_{i-2} \cdots b_{1}\left[L \xi_{2} \mathrm{~d} t+\phi_{[1, \sigma(t)]} \mathrm{d} t+\psi_{[1, \sigma(t)]}^{T} \mathrm{~d} \omega\right]
\end{gathered}
$$

where

$$
\begin{aligned}
d_{i, 1}= & -b_{i-1} \cdots b_{2} b_{1} b_{1}, \\
d_{i, j}= & b_{i-1} \cdots b_{j} b_{j-1}-b_{i-1} \cdots b_{j} b_{j}, \quad 2 \leq j \leq i-1, \\
d_{i, i}= & b_{i-1}, \\
\tilde{d}_{i}= & a_{i}+b_{i-1} b_{i-2} \cdots b_{2} a_{2}+b_{i-1} \cdots b_{3} a_{3}+\cdots \\
& +b_{i-1} a_{i-1} .
\end{aligned}
$$

Now, we have finished the design step $i-1$, and $\alpha_{i-1}$ is chosen as follows:

$$
\begin{aligned}
\alpha_{i-1}= & -L b_{i-1} \varepsilon_{i-1}, \\
b_{i-1}= & n+4 n C_{e}+\frac{5}{4}+\frac{\tilde{d}_{i-1}^{2}}{4}+\frac{1}{4} d_{i-1,1}^{2}+\cdots \\
& +\frac{1}{4} d_{i-1, i-1}^{2}+\frac{1}{4}\left(b_{i-2} \cdots b_{1}\right)^{2} \\
& +\frac{1}{4}\left(b_{i-2} \cdots b_{1}\right)^{2} \tau_{1}^{2}+d_{i-1, i-1} .
\end{aligned}
$$

Let $V_{i-1}=V_{i-2}+\left(1 / 2 L^{2 i-4}\right) \varepsilon_{i-1}^{2}$, such that

$$
\begin{aligned}
& \mathscr{L} V_{i-1} \leq-\left[\delta L-2 \delta\|P\|^{2}-2 n C_{e}-(i-1) L\right]\|\xi\|^{2} \\
& +2 n C_{e}\left(\frac{\left|\widehat{z}_{i+1}\right|^{2}}{L^{2 i}}+\cdots+\frac{\left|\widehat{z}_{n}\right|^{2}}{L^{2 n-2}}\right) \\
& -\{[2 n-2(i-1)+3] L \\
& \left.-\sum_{j=1}^{i-2} \frac{\left(b_{j} \cdots b_{1}\right)^{2} \tau_{2}^{2}}{2}-4 n C_{e} b_{1}^{2}\right\} \varepsilon_{1}^{2}-\sum_{j=2}^{i-1} \frac{1}{L^{2 j-2}} \\
& +\left[(n+j-i+1) L-4 n C_{e} b_{j}^{2}\right] \varepsilon_{j}^{2}+4 n c_{e} \frac{\varepsilon_{i}^{2}}{L^{2 i-3}} \\
& +\frac{\varepsilon_{i}^{2}}{4 L^{2 i-3}} .
\end{aligned}
$$

Defining $V_{i}=V_{i-1}+\left(1 / 2 L^{2 i-2}\right) \varepsilon_{i}^{2}$, we have

$$
\begin{aligned}
\mathscr{L} V_{i} & \leq-\left[\delta L-2 \delta\|P\|^{2}-2 n C_{e}-(i-1) L\right]\|\xi\|^{2} \\
+ & 2 n C_{e}\left(\frac{\left|\widehat{z}_{i+1}\right|^{2}}{L^{2 i}}+\cdots+\frac{\left|\widehat{z}_{n}\right|^{2}}{L^{2 n-2}}\right) \\
& -\left[[2 n-2(i-1)+3] L-\sum_{j=1}^{i-2} \frac{\left(b_{j} \cdots b_{1}\right)^{2} \tau_{2}^{2}}{2}\right. \\
& \left.-4 n C_{e} b_{1}^{2}\right] \varepsilon_{1}^{2} \\
& -\sum_{j=2}^{i-1} \frac{1}{L^{2 j-2}}\left[(n+j-i+1) L-4 n C_{e} b_{j}^{2}\right] \varepsilon_{j}^{2}+4 n C_{e} \\
& +\frac{\varepsilon_{i}^{2}}{L^{2 i-3}}+\frac{1}{4 L^{2 i-3}} \varepsilon_{i}^{2}+\frac{1}{L^{2 i-2}} \varepsilon_{i}\left[\varepsilon_{i+1}+\alpha_{i}+L^{i} \tilde{d}_{i} \xi_{1}\right. \\
+ & L^{i} d_{i, 1} \varepsilon_{1}+L^{i-1} d_{i, 2} \varepsilon_{2}+\cdots+L^{2} d_{i, i-1} \varepsilon_{i-1, p}+L d_{i, i} \varepsilon_{i} \\
+ & \left.L^{i-1} b_{i-1} b_{i-2} \cdots b_{1}\left(L \xi_{2}+\phi_{[1, \sigma(t)]}\right)\right] \\
+ & \frac{1}{2 L^{2 i-2}}\left(L^{i-1} b_{i-1} b_{i-2} \cdots b_{1}\right)^{2} \operatorname{Tr}\left[\psi_{[1, \sigma(t)]} \psi_{[1, \sigma(t)]}^{T}\right]
\end{aligned}
$$

By Lemma 2.1 in [28], one gets

$$
\begin{aligned}
& 2 n C_{e} \frac{\left|\widehat{z}_{i+1}\right|^{2}}{L^{2 i}} \leq 4 n C_{e} \frac{\varepsilon_{i+1}^{2}}{L^{2 i-1}}+4 n C_{e} \frac{\alpha_{i}^{2}}{L^{2 i}}, \\
& \frac{1}{L^{2 i-2}} \varepsilon_{i} \varepsilon_{i+1} \leq \frac{1}{4 L^{2 i-1}} \varepsilon_{i+1}^{2}+\frac{1}{L^{2 i-3}} \varepsilon_{i}^{2}, \\
& \frac{\widetilde{d}_{i}}{L^{i-2}} \varepsilon_{i} \xi_{1} \leq \frac{\widetilde{d}_{i}^{2}}{4 L^{2 i-3}} \varepsilon_{i}^{2}+L \xi_{1}^{2}, \\
& \frac{d_{i, 1}}{L^{i-2}} \varepsilon_{i} \varepsilon_{1} \leq \frac{d_{i, 1}^{2}}{4 L^{2 i-3}} \varepsilon_{i}^{2}+L \varepsilon_{1}^{2}, \\
& \frac{d_{i, i-1}}{L^{2 i-4}} \varepsilon_{i} \varepsilon_{i-1} \leq \frac{d_{i, i-1}^{2}}{4 L^{2 i-3}} \varepsilon_{i}^{2}+\frac{1}{L^{2 i-5}} \varepsilon_{i-1}^{2}, \\
& \frac{1}{L^{i-2}} b_{i-1} b_{i-2} \cdots b_{1} \varepsilon_{i} \xi_{2} \leq \frac{\left(b_{i-1} b_{i-2} \cdots b_{1}\right)^{2}}{4 L^{2 i-3}} \varepsilon_{i}^{2}+L \xi_{2}^{2},
\end{aligned}
$$




$$
\begin{aligned}
& \frac{1}{L^{i-1}} b_{i-1} b_{i-2} \cdots b_{1} \varepsilon_{i} \phi_{1 p} \leq \frac{\left(b_{i-1} b_{i-2} \cdots b_{1} \tau_{1}\right)^{2}}{4 L^{2 i-3}} \varepsilon_{i}^{2}+L \varepsilon_{1}^{2}, \\
& \frac{1}{2 L^{2 i-2}}\left(L^{i-1} b_{i-1} b_{i-2} \cdots b_{1}\right)^{2} \operatorname{Tr}\left\{\psi_{[1, \sigma(t)]} \psi_{[1, \sigma(t)]}^{T}\right\} \\
& \quad \leq \frac{\left(b_{i-1} b_{i-2} \cdots b_{1}\right)^{2}}{2} \tau_{2}^{2} \varepsilon_{1}^{2} .
\end{aligned}
$$

Choosing $\alpha_{i}$ as

$$
\begin{aligned}
\alpha_{i}= & -L b_{i} \varepsilon_{i}, \\
b_{i}= & +4 n C_{e}+\frac{5}{4}+\frac{\tilde{d}_{i}^{2}}{4}+\frac{1}{4} d_{i, 1}^{2}+\cdots+\frac{1}{4} d_{i, i-1}^{2} \\
& +\frac{1}{4}\left(b_{i-1} \cdots b_{1}\right)^{2}+\frac{1}{4}\left(b_{i-1} \cdots b_{1}\right)^{2} \tau_{1}^{2}+d_{i, i},
\end{aligned}
$$

one has

$$
\begin{aligned}
\mathscr{L} V_{i} & \leq-\left[\delta L-2 \delta\|P\|^{2}-2 n C_{e}-i L\right]\|\xi\|^{2} \\
& +2 n C_{e}\left(\frac{\left|\widehat{z}_{i+2}\right|^{2}}{L^{2 i+2}}+\cdots+\frac{\left|\widehat{z}_{n}\right|^{2}}{L^{2 n-2}}\right) \\
& -\left\{[2 n-2 i+3] L-\sum_{j=1}^{i-1} \frac{\left(b_{j} \cdots b_{1}\right)^{2} \tau_{2}^{2}}{2}-4 n C_{e} b_{1}^{2}\right\} \\
\cdot & \varepsilon_{1}^{2}-\sum_{j=2}^{i} \frac{1}{L^{2 j-2}}\left[(n+j-i) L-4 n C_{e} b_{j}^{2}\right] \varepsilon_{j}^{2}+4 n C_{1} \\
& \frac{\varepsilon_{i+1}^{2}}{L^{2 i-1}}+\frac{1}{4 L^{2 i-1}} \varepsilon_{i+1}^{2} .
\end{aligned}
$$

Step $n$. Letting $V_{n}=V_{n-1}+\left(1 / 2 L^{2 n-2}\right) \varepsilon_{n}^{2}$ and choosing

$$
\begin{aligned}
u= & -L b_{n} \varepsilon_{n}, \\
b_{n}= & n+4 n C_{e}+\frac{5}{4}+\frac{\widetilde{d}_{n}^{2}}{4}+\frac{d_{n, 1}^{2}}{4}+\cdots+\frac{d_{n, n-1}^{2}}{4} \\
& +\frac{\left(b_{n-1} \cdots b_{1}\right)^{2}}{4}+\frac{\left(b_{n-1} \cdots b_{1}\right)^{2} \tau_{1}^{2}}{4}+d_{n, n}
\end{aligned}
$$

one gets

$$
\begin{aligned}
\mathscr{L} V_{n} & \leq-h_{0}\|\xi\|^{2}-h_{1} \varepsilon_{1}^{2}-\sum_{j=2}^{n-1} h_{j} \frac{1}{L^{2 j-2}} \varepsilon_{j}^{2}-h_{n} \frac{1}{L^{2 n-3}} \varepsilon_{n}^{2} \\
& \leq-\tilde{h} V_{n},
\end{aligned}
$$

where $\widetilde{h}=\min \left\{h_{0} / \delta, 2 h_{1}, \ldots, 2 h_{n}\right\}$ and

$$
\begin{aligned}
& h_{0}=\delta L-2 \delta\|P\|^{2}-2 n C_{e}-n L \geq 0, \\
& h_{1}=3 L-\sum_{j=1}^{n-1} \frac{\left(b_{j} \cdots b_{1}\right)^{2} \tau_{2}^{2}}{2}-4 n C_{e} b_{1}^{2} \geq 0, \\
& h_{j}=j L-4 n C_{e} b_{j}^{2} \geq 0, \quad j=2, \ldots, n-1, \\
& h_{n}=n \geq 0, \quad \delta>n .
\end{aligned}
$$

Remark 10. From (20), one has that $b_{1}$ do not contain $L$ under arbitrary switching. From (28), one has that all $b_{i}$ do not contain $L$ under the designed controllers.

Remark 11. For example, with $n=3$, by (41) in [25] and (32), we have

$$
\begin{aligned}
& h_{0}=\delta\left(L-2\|P\|^{2}-\|B\|^{2}\right)-6 C_{e}-3 L \geq 0, \\
& h_{1}=6 L-4 L-2 \frac{\eta_{0}^{2}}{L}-\frac{\left(b_{2} b_{1}\right)^{2} \tau_{2}^{2}}{2}-12 C_{e} b_{1}^{2} \geq 0, \\
& h_{2}=2 L-\frac{\eta_{0}^{2}}{L}-12 C_{e} b_{2}^{2} \geq 0, \\
& h_{3}=3 \geq 0, \quad \delta>3, \\
& h_{0}=\delta\left(L-2\|P\|^{2}\right)-6 C_{e}-3 L \geq 0, \\
& h_{1}=7 L-4 L-\sum_{j=1}^{2} \frac{\left(b_{j} \cdots b_{1}\right)^{2} \tau_{2}^{2}}{2}-12 C_{e} b_{1}^{2} \geq 0, \\
& h_{2}=3 L-12 C_{e} b_{2}^{2} \geq 0, \\
& h_{3}=3 \geq 0, \quad \delta>3,
\end{aligned}
$$

respectively. It is easy to see that $h_{1}$ and $h_{2}$ in (33) are all quintic functions about $L$, but they are linear functions about $L$ in (34). So, the calculation of $\delta$ and $L$ will be more simple with the method in this manuscript.

Choose $V=V_{0}+V_{n}$, which together with (5) and (31) result in

$$
\mathscr{L} V \leq-h V
$$

where $h=\min \{\lambda, \widetilde{h}\}$.

Theorem 12. For system (1b), the closed-loop system with controller (30) is asymptotically stable in probability.

3.3. Switching Control. In the above two subsections, we give the controllers $u_{0}$ and $u$ with $x_{0}\left(t_{0}\right) \neq 0$ as (4) and (30). Now, we turn to the case of $x_{0}\left(t_{0}\right)=0$. If $x_{0}\left(t_{0}\right)=0$, we firstly choose constant control $u_{0}=u_{0}^{*} \neq 0$. Secondly, there will exist a time $t_{s}^{*}>0$ such that $x_{0}\left(t_{s}^{*}\right) \neq 0$. After that, controllers $u_{0}$ and $u$ as (4) and (30) can be applied.

Theorem 13. If we apply the above switching procedure, systems (1a) and (1b) will be asymptotically stabilized in probability.

\section{A Simulation Example}

Consider systems (1a) and (1b) with $\sigma:[0,+\infty) \rightarrow\{1,2\}$ and $g_{[0,1]}=c_{1} x_{0} \cos \left(x_{0}\right), g_{[0,2]}=c_{1} x_{0} \sin \left(x_{0}\right), f_{[1,1]}=$ $c_{1} x_{1} \cos ^{2}\left(x_{1}\right), f_{[1,2]}=c_{1} x_{1} \cos \left(x_{1}\right), g_{[1,1]}=c_{1} x_{1} \sin ^{2}\left(x_{1}\right)$, $g_{[1,2]}=c_{1} x_{1} \sin \left(x_{1}\right), f_{[2,1]}=f_{[2,2]}=0, g_{[2,1]}=c_{1} x_{2} \sin ^{2}\left(x_{2}\right)$, $g_{[2,2]}=c_{1} x_{2} \sin \left(x_{2}\right)$, and $c_{1}=0.001$.

From (8), we have $\phi_{[1,1]}=\eta_{0} z_{1}+c_{1} z_{1} \cos ^{2}\left(x_{1}\right)+$ $c_{1}^{2} z_{1} \cos ^{2}\left(x_{0}\right)-c_{1}^{2} z_{1} \sin ^{2}\left(x_{1}\right) \cos \left(x_{0}\right), \phi_{[2,1]}=0, \psi_{[1,1]}=$ 

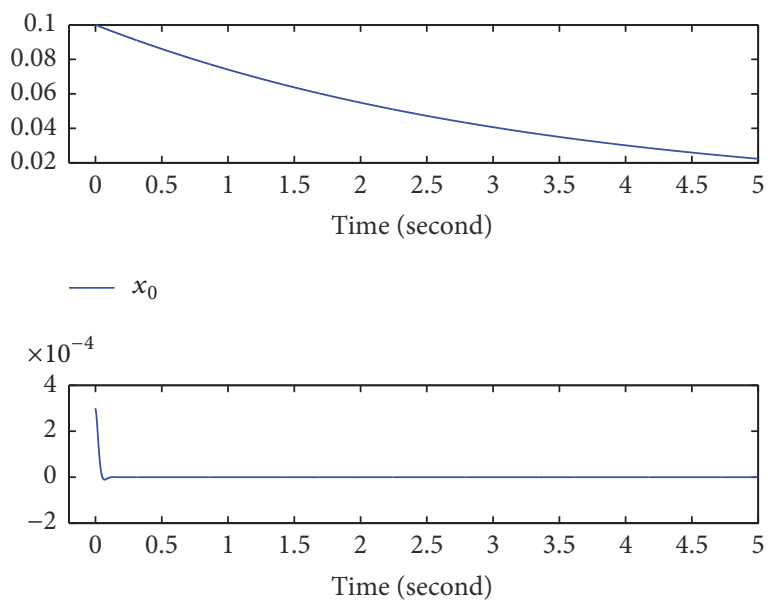

$-x_{1}$

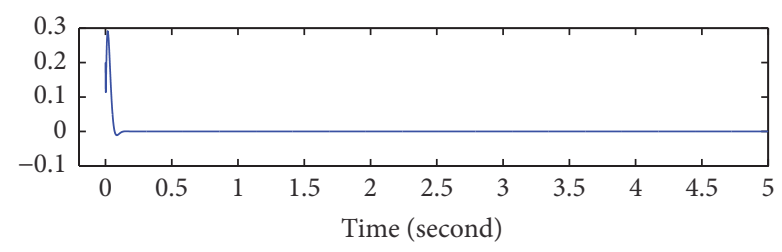

$-x_{2}$

FIGURE 1: The responses of states $x_{0}, x_{1}$, and $x_{2}$.

$c_{1} z_{1} \sin ^{2}\left(x_{1}\right)-c_{1} z_{1} \cos \left(x_{0}\right), \psi_{[2,1]}=c_{1} z_{2} \sin ^{2}\left(z_{2}\right), \phi_{[1,2]}=$ $\eta_{0} z_{1}+c_{1} z_{1} \cos ^{2}\left(x_{1}\right)+c_{1}^{2} z_{1} \sin ^{2}\left(x_{0}\right)-c_{1}^{2} z_{1} \sin \left(x_{1}\right) \sin \left(x_{0}\right)$, $\phi_{[2,2]}=0, \psi_{[1,2]}=c_{1} z_{1} \sin \left(x_{1}\right)-c_{1} z_{1} \sin \left(x_{0}\right)$, and $\psi_{[2,2]}=$ $c_{1} z_{2} \sin \left(z_{2}\right)$.

Letting $\eta_{0}=0.3$, by Proposition 8 , we have $\tau_{1}=0.3023$ and $\tau_{2}=0.001$. Choosing $a_{1}=0.75$ and $a_{2}=1.25$, then $\lambda_{\text {max }}(P)=2$ and $\|P\|=2$.

From (20) and (30), one gets

$$
\begin{aligned}
b_{1}= & 5+4 C_{e}+1+\frac{1}{4}+\tau_{1}+\frac{1}{2} \tau_{2}^{2}, \\
b_{2}= & 2+8 C_{e}+1+\frac{1}{4}+\frac{\left(a_{2}+b_{1} a_{1}\right)^{2}}{4}+\frac{b_{1}^{2}}{4}+b_{1}+\frac{b_{1}^{2}}{4} \\
& +\frac{b_{1}^{2} \tau_{1}^{2}}{4}, \\
u= & -L b_{2} \widehat{z}_{2}-L^{2} b_{2} b_{1} z_{1},
\end{aligned}
$$

where $C_{e}=0.4131 \delta$. From (32) and (35), we have

$$
\begin{aligned}
& h_{0}=(\delta-2) L-2 \delta\|P\|^{2}-4 C_{e} \geq 0, \\
& h_{1}=2 L-\frac{b_{1}^{2} \tau_{2}^{2}}{2}-8 C_{e} b_{1}^{2} \geq 0, \\
& h_{2}=2 \geq 0, \quad \delta>2 .
\end{aligned}
$$

Solving the above inequalities, one has $\delta=2.26$ and $L=76$, which means $h_{0}=0.0277, h_{1}=5.5526$, and $\mathscr{L} V \leq 0$.

If we choose initial values $x_{0}(0)=0.1, x_{1}(0)=0.0003$, $x_{2}(0)=0.2, \widehat{z}_{1}(0)=-0.001$, and $\widehat{z}_{2}(0)=7.5$, responses of systems are as in Figures 1, 2, and 3.

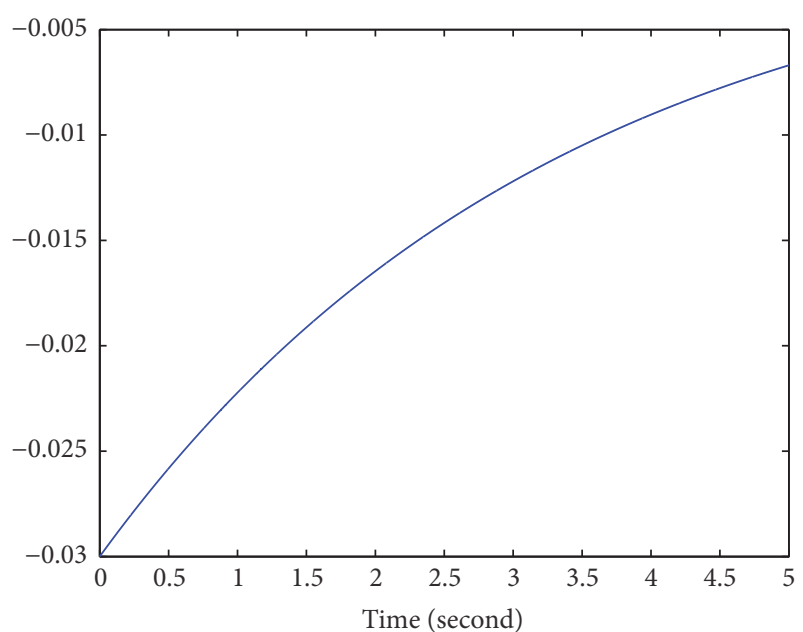

- $u_{0}$

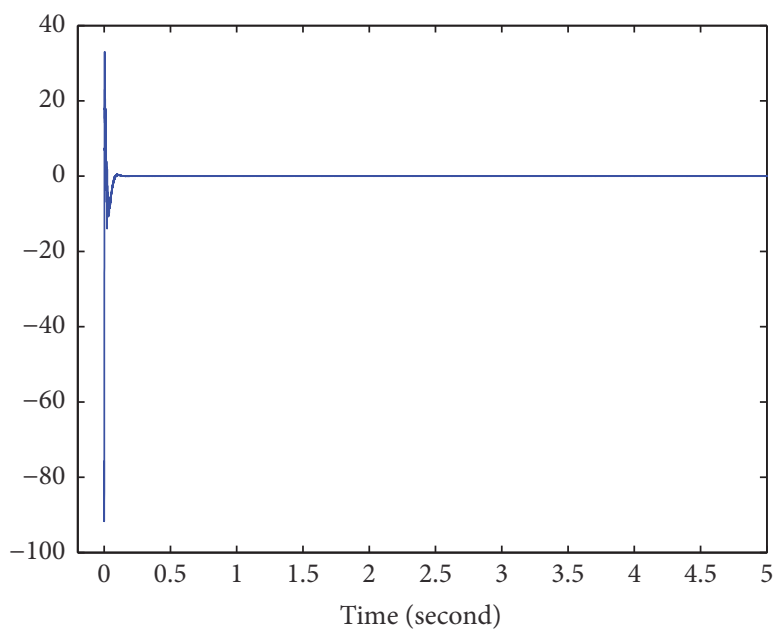

FIGURE 2: The responses of controllers $u_{0}$ and $u$.

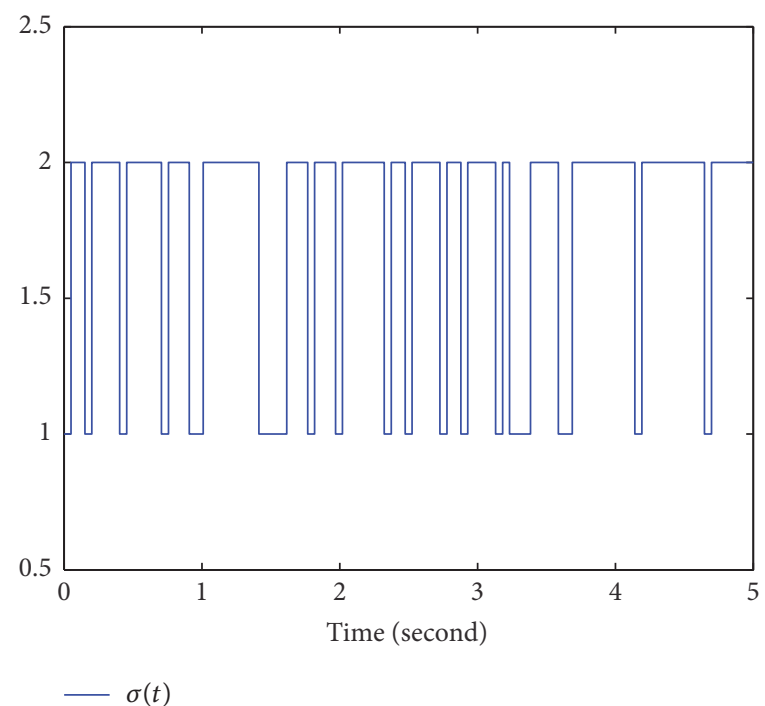

FIGURE 3: The responses of switching signals $\sigma(t)$. 
Remark 14. From the above example, the observer we adopted is the same as that in [27], but it can simplify the calculation of $\delta$ and $L$ compared with the observer in [25].

\section{Conclusions}

The output feedback stabilization for SNSs under arbitrary switching is discussed. We proposed an observer which is different from that in [25]. $b_{i}$ of the designed output feedback stabilizing control laws do not contain the gain parameter. We will give some new results, for example, how to design an adaptive controller with the method LMI based on results in $[29,30]$.

\section{Conflicts of Interest}

The authors declare that they have no conflicts of interest.

\section{Acknowledgments}

This work was partially supported by the National Natural Science Foundation of China (nos. 61503262 and 61374040), the Foundation for High-Level Talents of Hebei Province of China (nos. A2016001137 and A2016001144), and the Natural Science Foundation of Henan Educational Committee of China (no. 17A110027).

\section{References}

[1] J. Chen and C. Lin, "Static output feedback control for discretetime switched systems via improved path-following method," Discrete Dynamics in Nature and Society, vol. 2015, Article ID 869012, 8 pages, 2015.

[2] H. Sun, Q. Lu, and L. Hou, "Composite Adaptive Antidisturbance Control for Discrete-Time Switched System," Discrete Dynamics in Nature and Society, vol. 2016, Article ID 4269725, 9 pages, 2016.

[3] X. Zhao, P. Shi, Y. Yin, and S. . Nguang, "New results on stability of slowly switched systems: a multiple discontinuous Lyapunov function approach," Institute of Electrical and Electronics Engineers Transactions on Automatic Control, vol. 62, no. 7, pp. 35023509, 2017.

[4] R. Ma and J. Zhao, "Backstepping design for global stabilization of switched nonlinear systems in lower triangular form under arbitrary switchings," Automatica, vol. 46, no. 11, pp. 1819-1823, 2010.

[5] M. Hou, F. Fu, and G. Duan, "Global stabilization of switched stochastic nonlinear systems in strict-feedback form under arbitrary switchings," Automatica, vol. 49, no. 8, pp. 2571-2575, 2013.

[6] L. Wu, R. Yang, P. Shi, and X. Su, "Stability analysis and stabilization of 2-D switched systems under arbitrary and restricted switchings," Automatica, vol. 59, article no. 6426, pp. 206-215, 2015.

[7] C. Y. Wang and X. H. Jiao, "Adaptive control under arbitrary switching for a class of switched nonlinear systems with nonlinear parameterisation," International Journal of Control, vol. 88, no. 10, pp. 2044-2054, 2015.

[8] T. Jiao, S. Xu, Y. Li, and Z. Li, "Adaptive stabilisation of random systems with arbitrary switchings," IET Control Theory \& Applications, vol. 9, no. 18, pp. 2634-2640, 2015.
[9] Y.-J. Liang, R. Ma, M. Wang, and J. Fu, "Global finite-time stabilisation of a class of switched nonlinear systems," International Journal of Systems Science, vol. 46, no. 16, pp. 2897-2904, 2015.

[10] S. Huang and Z. Xiang, "Finite-time stabilization of a class of switched stochastic nonlinear systems under arbitrary switching," International Journal of Robust and Nonlinear Control, vol. 26, no. 10, pp. 2136-2152, 2016.

[11] L. Liu, X.-D. Zhao, B. Niu, H.-Q. Wang, and X.-J. Xie, “Global output-feedback stabilisation of switched stochastic non-linear time-delay systems under arbitrary switchings," IET Control Theory \& Applications, vol. 9, no. 2, pp. 283-292, 2015.

[12] X.-L. Liang, M.-Z. Hou, and G.-R. Duan, "Output feedback stabilization of switched stochastic nonlinear systems under arbitrary switchings," International Journal of Automation and Computing, vol. 10, no. 6, pp. 571-577, 2013.

[13] D. Zhang, C. Wang, J. Qiu, and H. Chen, "State-feedback stabilisation for stochastic non-holonomic systems with Markovian switching," International Journal of Modelling, Identification and Control, vol. 16, no. 3, pp. 221-228, 2012.

[14] Q. Du, C. Wang, G. Wang, and D. Zhang, "State-feedback stabilization for stochastic high-order nonholonomic systems with Markovian switching," Nonlinear Analysis: Hybrid Systems, vol. 18, pp. 1-14, 2015.

[15] J. Wang, H. Gao, and H. Li, "Adaptive robust control of nonholonomic systems with stochastic disturbances," Science China Information Sciences, vol. 49, no. 2, pp. 189-207, 2006.

[16] D. Zhang, C. Wang, and H. Chen, "Adaptive state-feedback stabilization for stochastic nonholonomic chained systems," Control Theory Applications, vol. 29, no. 11, pp. 1479-1487, 2012.

[17] D. Zhang, C. Wang, H. Chen, F. Yang, and J. Du, "Adaptive stabilization of stochastic non-holonomic systems with nonhomogeneous uncertainties," Transactions of the Institute of Measurement and Control, vol. 35, no. 5, pp. 648-663, 2013.

[18] F. Gao and F. Yuan, "Adaptive stabilization of stochastic nonholonomic systems with nonlinear parameterization," Applied Mathematics and Computation, vol. 219, no. 16, pp. 8676-8686, 2013.

[19] F. Gao and F. Yuan, "Finite-time stabilization of stochastic nonholonomic systems and its application to mobile robot," Abstract and Applied Analysis, vol. 2012, Article ID 361269, 18 pages, 2012.

[20] F. Gao, F. Yuan, and Y. Wu, "State-feedback stabilisation for stochastic non-holonomic systems with time-varying delays," IET Control Theory \& Applications, vol. 6, no. 17, pp. 2593-2600, 2012.

[21] Z. J. Wu and Y. H. Liu, "Stochastic stabilization of nonholonomic mobile robot with heading-angle-dependent disturbance," Mathematical Problems in Engineering, vol. 2012, Article ID 870498, 17 pages, 2012.

[22] W. Feng, Q. Sun, Z. Cao, D. Zhang, and H. Chen, "Adaptive state-feedback stabilization for stochastic nonholonomic mobile robots with unknown parameters," Discrete Dynamics in Nature and Society, vol. 2013, Article ID 808249, 9 pages, 2013.

[23] X. Zheng and Y. Wu, "Output feedback stabilization of stochastic nonholonomic systems," in Proceedings of the 8th World Congress on Intelligent Control and Automation, WCICA, pp. 2091-2096, Jinan, China, July 2010.

[24] Y.-L. Liu and Y.-Q. Wu, "Output feedback control for stochastic nonholonomic systems with growth rate restriction," Asian Journal of Control, vol. 13, no. 1, pp. 177-185, 2011. 
[25] D. Zhang, C. Wang, G. Wei, and H. Chen, "Output feedback stabilization for stochastic nonholonomic systems with nonlinear drifts and Markovian switching," Asian Journal of Control, vol. 16, no. 6, pp. 1679-1692, 2014.

[26] M. Krstic' and H. Deng, Stabilization of Nonlinear Uncertain Systems, Communications and Control Engineering, Springer, London, UK, 1998.

[27] S.-J. Liu and J.-F. Zhang, "Output-feedback control of a class of stochastic nonlinear systems with linearly bounded unmeasurable states," International Journal of Robust and Nonlinear Control, vol. 18, no. 6, pp. 665-687, 2008.

[28] W. Lin and C. Qian, "Adding one power integrator: a tool for global stabilization of high-order lower-triangular systems," Systems \& Control Letters, vol. 39, no. 5, pp. 339-351, 2000.

[29] Z. Zhang and S. Yu, "Global asymptotic stability for a class of complex-valued Cohen-Grossberg neural networks with time delays," Neurocomputing, vol. 171, pp. 1158-1166, 2016.

[30] Y.-P. Tian and K.-C. Cao, "An LMI design of tracking controllers for nonholonomic chained-form system," in Proceedings of the 2007 American Control Conference, ACC, pp. 4512-4517, USA, July 2007. 


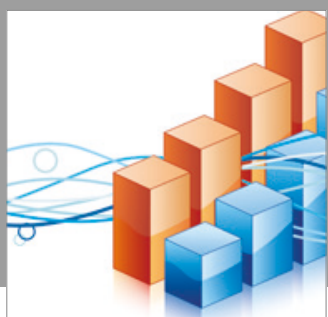

Advances in

Operations Research

vatersals

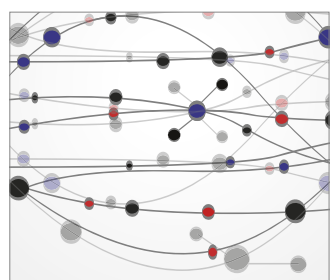

\section{The Scientific} World Journal
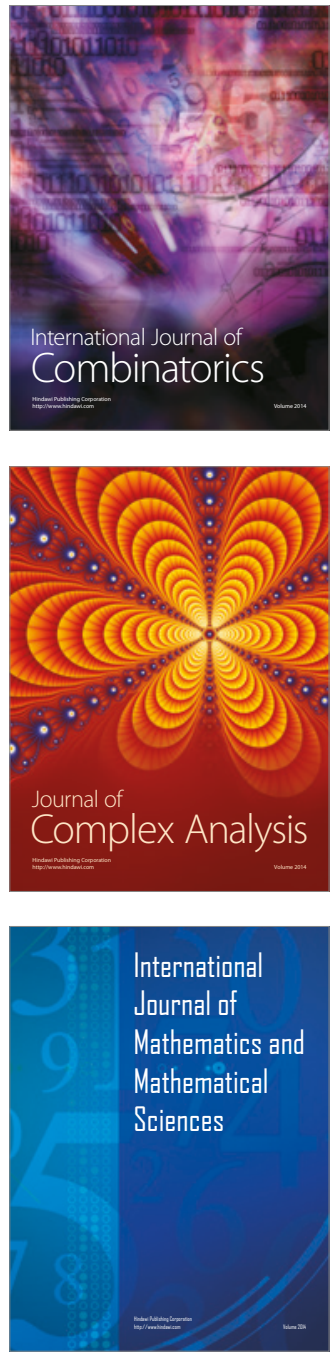
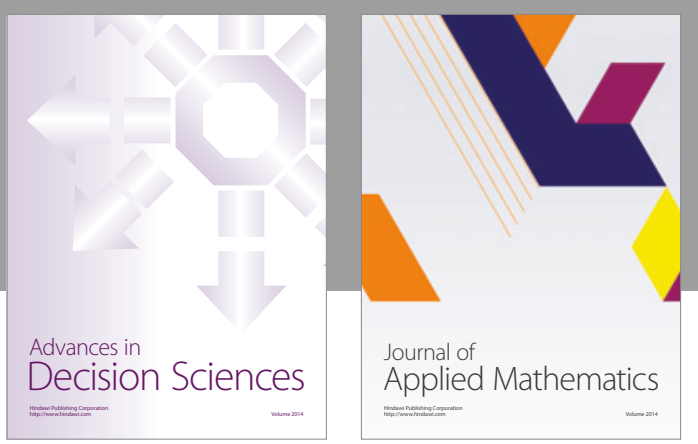

Algebra

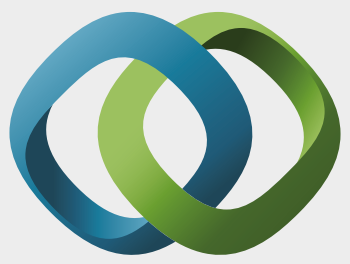

\section{Hindawi}

Submit your manuscripts at

https://www.hindawi.com
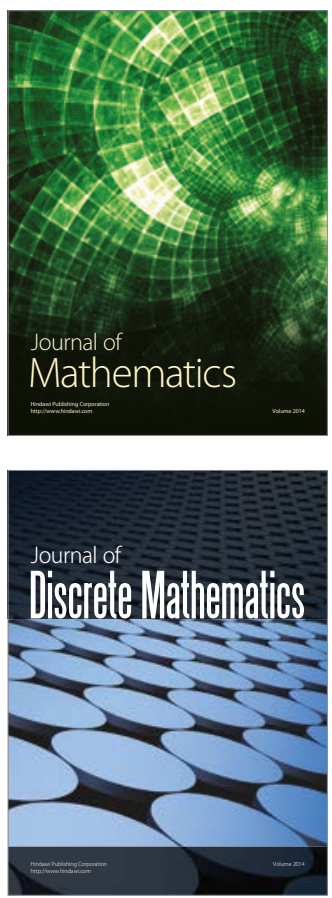

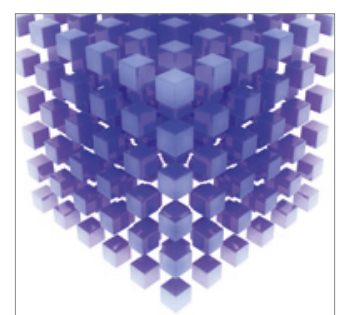

Mathematical Problems in Engineering
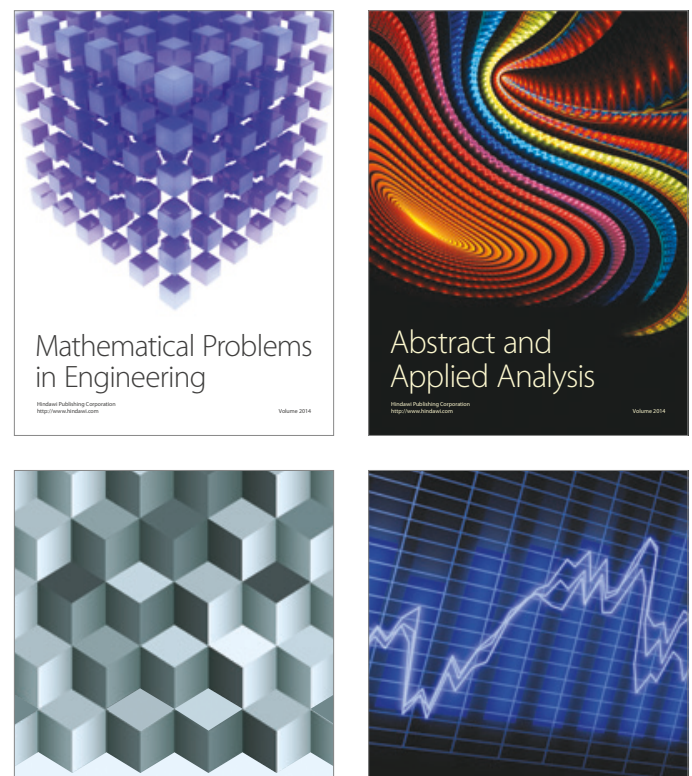

Journal of

Function Spaces

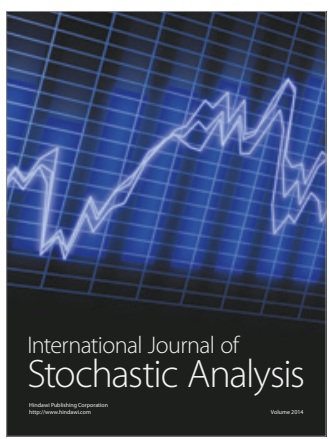

Probability and Statistics
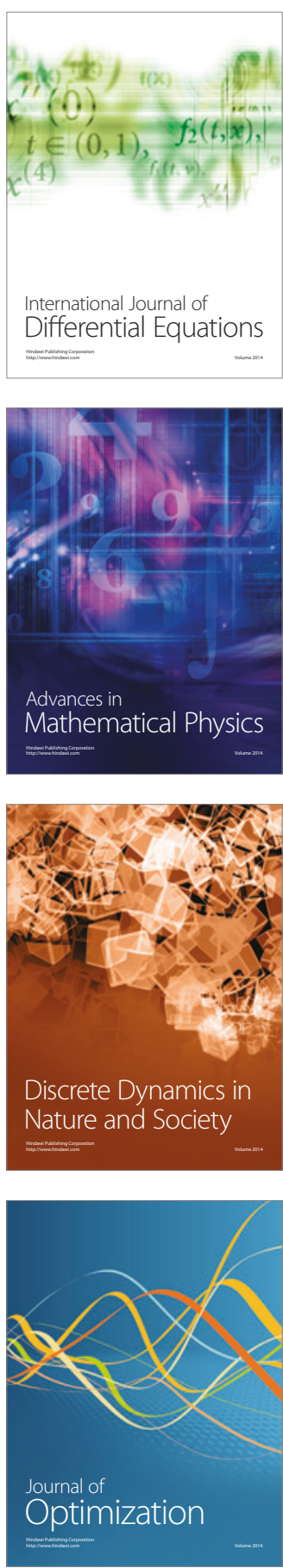